Planetary Systems in the Universe - Observation, Formation and Evolution

Proceedings IAU Symposium No. 202, (C2004 IAU

Alan Penny, Pawel Artymowicz, Anne-Marie Lagrange, \& Sara Russell, eds.

\title{
Search for Signatures of an Atmosphere of HD209458 b
}

\author{
H. Rauer
}

DLR, Inst. of Space Sensor Techn. and Plan. Expl., Berlin, Germany

A. Collier Cameron, J. Barnes

University of St. Andrews, United Kingdom

\section{A. W. Harris}

DLR, Inst. of Space Sensor Techn. and Plan. Expl., Berlin, Germany

\begin{abstract}
We investigate the prospects of detecting the presence of $\mathrm{Na} \mathrm{D}$ absorption features during transits of the planet of HD209458 by means of highresolution spectroscopy.
\end{abstract}

Photometric transit and radial velocity measurements of HD209458 (Charbonneau et al. 2000; Henry et al. 2000) provide the $\operatorname{mass}\left(\mathrm{M}_{p}=0.69 \mathrm{M}_{J}\right)$ and radius $\left(R_{p}=1.40 R_{J}\right)$ of its planet (Mazeh et al., 2000). These measurements confirm the gas-giant nature of the planet. Key parameters which determine the efficiency of heating and cooling processes since formation of the planet are the composition and opacity of the atmosphere and the level of cloud formation.

We are exploring the possibility of investigating the nature of extra-solar planet atmospheres via absorption features observed during transits. Planets not fully eclipsing their star, but with sufficiently high orbital inclination, may possess extended exospheres or magnetospheres which could be observed transiting across the stellar surface (Schneider et al. 1998; Rauer et al. 2000; Harris et al. 2000). Eclipsing planets, such as HD209458b, may allow the transparent part of their atmosphere to be investigated. Alkali metal absorptions may be important in determining the planet's albedo (Sudarsky et al., 2000). Models of transmission spectra of HD209458 (Seager and Sasselov, 2000) predict that optical depth unity is attained in the cores of $\mathrm{Na} \mathrm{I} \mathrm{D} \mathrm{lines} \mathrm{several} \mathrm{thousand}$ kilometers above the cloud deck which defines the limb of the planet. These lines appear as narrow absorptions of the order of 0.002 times the background flux.

We observed HD209458 on November 25, 1999, using the Utrecht Echelle Spectrograph on the $4.2 \mathrm{~m}$ William Herschel telescope at the Roque de los Muchachos Observatory on La Palma, covering a wavelength range of 4040 - $6840 \AA$. Orbital phases were calculated for an assumed time of transit at HJD $2451430.823 \pm 0.003$ (Charbonneau et al., 2000) and an orbital period of $3.524739 \pm 0.000014 \mathrm{~d}$ (Robichon \& Aranaon, 2000). Resulting mid-transit times were 20:49 UT. Our observations started at 21:23 UT (34 min after mid-transit) and continued until 23:09 UT (140 min after transit). 


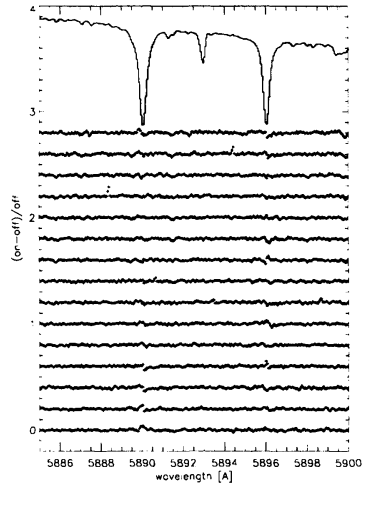

Fig. 1: The top of this figure shows the $\mathrm{Na} \mathrm{D}$ lines in the template spectrum. Below, a time series of residuals is shown. Time increases upward. Bottom line: 21:23 UT, top: 23:09 UT

We have concentrated on the search for Na I absorption in the planetary atmosphere. The data analysis procedure used is similar to the method used by Cameron et al. (1999). The spectra obtained out of transit were aligned and summed to form a template spectrum which represents the stellar spectrum without planetary signatures. The template, $T(\lambda)$, is used to approximate the stellar spectrum, $S(\lambda)$, in each of the on-transit spectra by scaling, shifting and compensating for small seeing-dependent changes in the line spread function using a Taylor expansion series:

$$
S(\lambda)=a_{0} T(\lambda)+a_{1} \frac{\partial T(\lambda)}{\partial \lambda}+a_{2} \frac{\partial^{2} T(\lambda)}{\partial \lambda^{2}} .
$$

The distorted template model is then subtracted from the observed flux spectra and the result divided by the template ((on-transit - off-transit)/offtransit). The residuals (Fig. 1) show enhanced noise in the cores of the sodium lines, but no signature of planetary sodium absorption.

The $\mathrm{Na}$ I doublet appears twice in the spectrum in two adjacent orders. To take advantage of this, we use a least-squares deconvolution technique (Donati et al., 1997) to stack up the absorption lines in the spectrum in an optimally weighted fashion. Figure 2 shows the trailed deconvolved $\mathrm{Na} \mathrm{D}$ line profile. The residuals, after subtracting the template model from each of the deconvolved spectra corresponding to the time-series shown in Fig. 1, are shown in the right part of Fig. 2.

The residuals in the 750 pixels shown here yield $\chi^{2}=643$, confirming that the errors are dominated by photon statistics. The expected width of the gaussian absorption profile due to the planet's atmosphere is 2 pixels. The RMS error in the individual pixel values is $6 \times 10^{-3}$ times the mean continuum level. In a single spectrum we could thus have detected a signal about 250 times fainter than the stellar continuum on a $1 \sigma$ level, if it were Doppler shifted completely off the line. In practice, however, the planet's absorption signature always appears within $15 \mathrm{~km} / \mathrm{sec}$ of the cores of the stellar lines, where the stellar flux is on average $1 / 3$ of the continuum level. This would yield a $1 \sigma$ detection of an absorption feature with an amplitude 140 times less than the background flux level in a single exposure. 

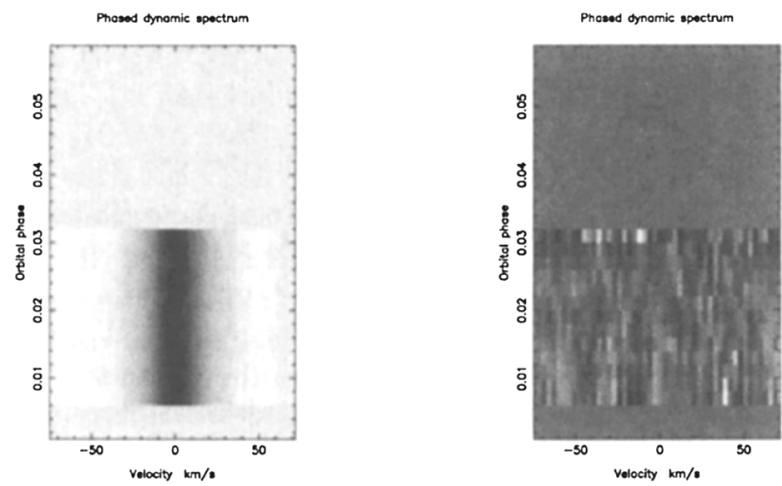

Fig. 2 Left: A trailed spectrum of the least-square deconvolved sodium D line profile. The greyscale covers 0 to 1.2 times the local continuum level. Right: residuals of least-square deconvolved profiles (time-series corresponding to Fig. 1) after subtracting the averaged least-square deconvolved template profile.

In order to detect a marginally-resolved atmospheric absorption feature with an amplitude 500 times smaller than the background flux (Seager 2000, personal communication) with $3 \sigma$ significance or better, we would require 120 such 5-minute exposures on the 4.2-m WHT+UES during transits, and a comparable number of spectra off-transit. This would require observations spanning 4 complete nights on which transits occur. We conclude, therefore, that the detection of $\mathrm{Na} \mathrm{D}$ absorption in the planet's atmosphere is feasible given a modest number of observed transits on a $4 \mathrm{~m}$-class telescope.

\section{References}

Cameron, A., Horne, K., Penny, A., \& James, D. 1999, Nature, 402, 751

Charbonneau, D., Brown, T., Latham, D., \& Mayor, M. 2000, ApJ, 529, L45

Donati, J.-F., Semel, M., Carter, B. et al. 1997, MNRAS, 291, 658

Harris, A. W., Rauer, H., Cameron, A. C. 2000, Disks, Planetesimals and Planets, ASP Conf. Ser., in press.

Henry, G., Marcy, G., Butler, R., Vogt, S. 2000, ApJ, 529, L41

Mazeh, T., Naef, D., Torres, G. et al. 1999, ApJ, 532, L55

Rauer, H., Bockelée-Morvan, D., Coustenis, A. et al. 2000, A\&A, 355, 573

Robichon, N., \& Aranaon, F. 2000, A\&A, 355, 295

Schneider, J., Rauer, H., Lasota, J. P. et al. 1998, ASP Conf. Ser. 134, Brown Dwarfs and Extrasolar Planets, eds. Rebolo, Martin, Zapatero, 241

Seager, S., \& Sasselov, D. D. 2000, ApJ, 537, 916

Sudarsky, D., Burrows, A., \& Pinto, P. 2000, ApJ, 538, 885 